\title{
Schreiben des Herrn Dr. W. Huggins an den Herausgeber.
}

Will you permit me to say in reference to Dr. Lohse's account of his photographic experiments, that I had overlooked the short statement of them in the vierteljahrsschrifte, and did not know that Dr. Lohse had made any experiments in this direction. If I had known of them it would have given me much pleasure to have referred to Dr. Eohse in my paper on photographing the solar corona.

Upper Tulse Hill. S. W. 1883 Jan. 30.

Willliam Huggins.

\section{Observations de la grande comète 1882 II à Plonsk.}

(Micromètre circulaire de la lunette de $162 \mathrm{~mm}$. d'objectif.)

Les observations suivantes ne peuvent pas prétendre a l'exactitude exigée et -doivent être prises avec le poids convenable, malgré qu'elles étaient faites avec toutes les précautions ordinaires et corrigées de la réfraction et du mouvement propre. La basse position de l'astre et son aspect apparent en sont la cause.

Lorsque j'aperçus la comète le 5 Janvier $188_{3}$, après quelques semaines de nuages continuels, elle présentait l'image d'une ovale pâle et nebuleuse de 4 ' de longueur à peu près avec le milieu plus clair, sans noyau appréciable. La partie centrale plus claire était elle-nhême aussi oblongue et située hors du centre de la masse nébuleuse, plus près de la partie étroite de l'ovale tournée vers le soleil. Les pointées étaient dirigées autant que possible vers le centre de la partie brillante.

L'a suppression d'une décimale aussi bien dans $\Delta \alpha$, que dans $\Delta \delta$, me semble suffisamment justifiée par les susdites circonstances.

\begin{tabular}{|c|c|c|c|c|c|c|c|c|c|}
\hline I 883 & T.M. Plonsk & $\Delta \alpha$ & $\Delta \delta$ & Pass. & $\alpha$ app. & $\log p . \Delta$ & $\delta$ app. & $\log p . A$ & * \\
\hline nv. 5 & $12^{h} 3^{m} 6^{s} \mathrm{I}$ & $+6^{\mathrm{m}} 53^{\mathrm{s}} \cdot 5$ & $+8^{\prime} 15^{\prime \prime}$ & 6 & $7^{\mathrm{h}} \quad 0^{\mathrm{m}} 57^{\mathrm{s}} \cdot 4$ & 8.7 & $-28^{\circ} 40^{\prime} 44^{\prime \prime}$ & 0.942 & I \\
\hline 6 & $1221 \quad 13.0$ & +241.2 & -215 & 5 & $\begin{array}{lll}6 & 58 & 21.2\end{array}$ & 8.692 & $\begin{array}{lll}-28 & 28 & 5^{8}\end{array}$ & 0.9 & 2 \\
\hline I I & $\begin{array}{lll}1 & 37 & \mathbf{1} 8.5\end{array}$ & $-35^{2.5}$ & -029 & 7 & $\begin{array}{lll}6 & 4^{6} & 2.6\end{array}$ & 8.440 & -272525 & $0.94 \mathrm{I}$ & 3 \\
\hline 12 & $\begin{array}{lll}1 & 25 & 0.4\end{array}$ & - I 41.8 & $+\circ 37$ & 8 & $\begin{array}{lll}6 & 43 & 45 \cdot 7\end{array}$ & 8.223 & -27 I I 32 & $0.94 \mathrm{I}$ & 4 \\
\hline 13 & I I $35 \quad 38.5$ & -650.4 & -80 & 7 & $64 \mathrm{I} 3$ 3. I & 8.666 & $-26 \quad 56 \quad 55$ & 0.940 & 5 \\
\hline 26 & $\begin{array}{lll}9 & 16 & 29.9\end{array}$ & + I 45.4 & $-\times 47$ & 6 & 6 I 731.5 & $8.8 \mathrm{I} \mathrm{2n}_{\mathrm{n}}$ & -23376 & 0.933 & 6 \\
\hline
\end{tabular}

Etoiles de comparaison.

\begin{tabular}{|c|c|c|c|c|c|}
\hline$*$ & $\mathrm{Gr}$. & $\alpha \quad 8883.0$ & $\delta 1883.0$ & Red. in l. app. & Autorité \\
\hline $\mathbf{I}$ & 2.1 & $6^{\mathrm{h}} 54^{\mathrm{m}} \quad \times \frac{5}{5}$ & $-28^{\circ} 48^{\prime} 49^{\prime \prime} 8$ & $+2.28-9.8$ & F. Cat. $\varepsilon$ Can. maj. \\
\hline 2 & 8.5 & $\begin{array}{lll}6 & 55 & 37.74\end{array}$ & $\begin{array}{lll}-28 & 26 & 32.9\end{array}$ & $+2.28-10.0$ & Anon. comp. avec $a$ \\
\hline 3 & 8.0 & 64952.87 & $\begin{array}{lll}-27 & 24 & 44.4\end{array}$ & $+2.29-1 \mathrm{r} .6$ & Yarnall 2799 \\
\hline 4 & $7 \cdot 5$ & $6.45 \quad 25.22$ & -27 II $\quad 57.4$ & $+2.28-12.0$ & $\gg \quad 2764$ \\
\hline 5 & 6.2 & $648 \times 8.28$ & $\begin{array}{lll}-26 & 48 & 43.4\end{array}$ & $+2.28-12.2$ & BB. VI. $6^{\mathrm{h}}$ I 65 \\
\hline 6 & 8.0 & $6 \times 544.01$ & $\begin{array}{lll}-23 & 35 & 3.6\end{array}$ & $+2.13-15.4$ & Yarnall 2608 \\
\hline$a$ & 6.9 & $\begin{array}{lll}6 & 56 & 5.04\end{array}$ & -28 I9 28.1 & $+2.28-10.1$ & $\gg \quad 2834$ \\
\hline
\end{tabular}

Plonsk 1883 Février.

Dr. Fedrzejewicz. 